hepatitis resulting in fatal outcome were detected in patients taking rifampicin, ethambutol, isoniazid and pyrazinamide. Anaphylactic shock was observed in patients with injected streptomycin. Skin and appendages disorders was the most common adverse effects reported. The result can be predictor of TB surveillance system to develop plan for TB patients surveillance.

\section{SP3-53 CHANGES IN THE NUMBER OF ORAL HEALTH-RELATED QUALITY OF LIFE IMPACTS IN ELDERLY PEOPLE}

doi:10.1136/jech.2011.1429760.53

F Andrade, ${ }^{*}$ M L Lebrão, J Santos, Y Duarte. University of São Paulo, São Paulo/São Paulo, Brazil

Objectives The aim of this study was to evaluate the pattern of changes in the number of oral-health-related-quality of life (OHRQoL) impacts and investigate its relationship with selfreported oral health measurements in a sample of communitydwelling elderly from São Paulo-Brazil.

Methods The sample consisted of 747, 65 and older people, enrolled in the Health, Well-being and Ageing cohort-study. OHRQoL negative-impacts were measured using the General Oral Health Index-GOHAI and calculated as the number of items reported as "always" or "often." Self-reported oral health measurements included: number of teeth lost and use of prosthesis. Changes scores were calculated by subtracting the number of impacts at baseline from the impacts at follow-up. A positive change score indicates an increment in the number of impacts, a negative score indicates a decrement and a change score of zero represents no change. Multinomial logistic regression analysis was used, estimating values for $\mathrm{OR}$ and a $95 \% \mathrm{CI}$. A design effect correction was made using the Stata survey command to analyse data coming from complex samples.

Results Most people had no change in the number of impacts and $21.80 \%$ had a decrement. Increment was related with tooth loss $\geq 16$ (OR 1.74), use of dental prosthesis (OR 0.54), schooling $\leq 3$ years (OR 1.65) and age (OR 1.03). Decrement was associated with tooth loss $\geq 16$ (OR 2.61), use of dental prosthesis (OR 0.52).

Conclusion Changes in OHRQoL impacts were significantly related with the number of teeth lost and use of prosthesis even after controlling for socioeconomic factors.

\section{SP3-54 MIDTERM CONSEQUENCES ON HEALTH OF THE EARTHOUAKE OF 6 APRIL 2009 IN L'AQUILA (ITALY), ASSESSED BY THE BEHAVIOURAL RISK FACTOR SURVEILLANCE SYSTEM PASSI}

doi:10.1136/jech.2011.1429760.54 disorder (PTSD) with the objective to establish a knowledge base for better informed public health decisions.

Methods From June to October 2010, 958 people living in earthquake area were interviewed by phone (response rate 95\%), using the properly modified PASSI questionnaire.

Results $92 \%$ of interviewees were in the earthquake area at time of event; $5 \%$ were injured; $10 \%$ knew an injured person, $40 \%$ knew a person killed by the earthquake among their relatives or friends, $35 \%$ had to leave their home due to severe damage, $45 \%$ reported economic losses and 15\% lost their job. $22 \%$ were displaced in a temporary public accommodation. $4 \%$ met the definition of PTSD and $16 \%$ of depression, higher for women and 50-69 aged years old. Conclusion The study provides an estimate of non-traumatic consequences of the earthquake, otherwise neglected but financially covered by the NHS, and is a useful tool for public health needs assessment and planning in case of a disaster.

\section{SP3-55 LAYERS OF COMPLEXITY IN INTERPRETING EFFECTIVENESS EVIDENCE}

doi:10.1136/jech.2011.1429760.55

${ }^{1}$ A Tannahill, ${ }^{*}$ M P Kelly. ${ }^{1}$ NHS Health Scotland, Glasgow, UK; ${ }^{2}$ National Institute for Health and Clinical Excellence, London, UK

Introduction There has been growing attention to using effectiveness evidence to guide public health and health improvement policies, strategies, programmes, and actions "on the ground." However, there has only been partial recognition of complications that have a material bearing on how such evidence is interpreted and translated into action. This paper aims to shed further light on such complications, capitalising on the authors' previous roles as "evidence originators" in academia and their more recently gained perspectives as "evidence interpreters" in national agencies.

Methods A particular randomised controlled trial in which the authors were directly involved-based on a workplace cardiovascular disease prevention programme-was revisited and used as an illustrative case study to elucidate important considerations in assessing and applying effectiveness evidence more generally.

Results Relatively obvious, and less obvious, complicating factors were identified in relation to defining the intervention, judging effectiveness, and transferability of findings. In addition, some "bigger picture" considerations were described, with individual interventions viewed as pieces in the health improvement "jigsaw" or as frames in the "movie" of ever-changing influences on population health.

Conclusion The layers of complexity uncovered in this work should be taken into account in designing, executing and reporting primary evaluative studies and reviews, in formulating recommendations for action, and in developing more fully fit-for-purpose approaches to evidence-informed public health and health improvement.

\section{SP3-56 TRENDS IN CARDIOVASCULAR RISK FACTORS IN THE UK, 1961-2011}

doi:10.1136/jech.2011.1429760.56 of L'Aquila, L'Aquila, Italy; ${ }^{3}$ Zadig, Roma, Italy; ${ }^{4}$ AUSL Pescara, Pescara, Italy; ${ }^{6}$ AUSL Teramo, Teramo, Italy; ${ }^{5}$ AUSL L'Aquila, Avezzano e Sulmona, L'Aquila, Italy; ${ }^{7}$ AUSL Lanciano-Vasto e Chieti, Chieti, Italy; ${ }^{8}$ AUSL Roma C, Roma, Italy

Intoduction 1 year after the earthquake in L'Aquila, all Abruzzo Local Health Units (LHU) with the University of L'Aquila, the Italian institute for health and the support of the Ministry of health, carried out a survey on midterm consequences of the earthquake on health. The analysis, based on the behavioural risk factors surveillance system PASSI, was aimed at assessing the prevalence of symptoms of depression and anxiety in adults, at monitoring health related behaviour and the prevalence of post-traumatic stress
Introduction Cardiovascular disease rates in the UK have been steadily declining over the past 50 years, mainly due to improvements in risk factors. This paper focuses on the behavioural risk factors and aims to document trends from 1961 to the present.

Methods We searched the peer-reviewed and grey literature from comparable estimates of prevalence of smoking, poor diet, alcohol consumption, physical inactivity and overweight and obesity, within the UK between 1961 and 2011. 